\title{
Acute Macular Neuro-retinopathy: a Rare Retinal Disorder, Presenting as Paracentral Scotoma
}

\author{
Royala Zaka', Yasir Khan ${ }^{2}$, Zaki-ud-Din Ahmed Sabri ${ }^{3}$ \\ ${ }^{1-3}$ Prevention of Blindness a Trust Based Hospital, Karachi - Pakistan
}

\begin{abstract}
Acute Macular Neuro-retinopathy (AMN) is a rare clinical entity. We present a case of 26 years old male who presented with one-week old history of sudden onset of decrease vision in left eye associated with paracentral scotomas. Dilated fundus examination of the left eye showed multiple reddish brownish petalloid para-foveal lesions with apex pointing toward the fovea. OCT showed hyper-reflective bands in the Outer Nuclear Layer and Outer Plexiform Layer along with disruption of ellipsoid zones. Amsler grid drawn by the patient and the visual field showed scotoma corresponding to the macular lesion. The cause turned out to be undiagnosed essential hypertension. Purpose of presenting this case is that High Definition Optical coherence tomography (SD-OCT) makes diagnosis of some rare conditions easy and fast for an ophthalmologist, that might be misdiagnosed or missed with conventional OCT and FFA imaging test.
\end{abstract}

Key Words: Acute Macular Neuro-retinopathy, Spectral Domain Optical Coherence Tomography, Paracentral Scotoma.

How to Cite this Article: Zaka R, Khan Y, Sabri ZDA. Acute Macular Neuro-retinopathy: a Rare Retinal Disorder, Presenting as Paracentral Scotoma. Pak J Ophthalmol. 2021, 37 (3): 327-329.

Doi: $10.36351 /$ pjo.v37i3.1225

\section{INTRODUCTION}

Bos and Deutman in 1975 were the first to describe Acute macular Neuro-retinopathy (AMN). ${ }^{1}$ Since then, one hundred and fifty six cases of AMN have been reported till 2016. ${ }^{2,3}$ Five cases of AMN, reportedly caused by non-ocular trauma, were studied on OCT first by Nentwich et al, that showed the defect in the outer retina supporting the Gillies et al work.,

Based on OCT findings, there are 2 types of AMN, type 1 the classic AMN points to hyperreflective bands in the Outer plexiform layer along with ellipsoid disruption and outer nuclear layer and type 2 AMN refers to hyper-reflective bands in the

Correspondence: Royala Zaka

Prevention of Blindness a Trust Based Hospital, KarachiPakistan

Email: drroyala@hotmail.com

Received: February 09, 2021

Accepted: April 28, 2021
Outer plexiform and inner nuclear layer. ${ }^{6}$ Deep retinal capillary plexus ischemia is thought to be associated with both types. ${ }^{7}$

We describe a case of AMN in a 26-year old male who presented with paracentral scotoma and on OCT it was diagnosed as AMN.

\section{Case Presentation}

A 26-year old man presented in eye out patient department with history of decrease vision in left eye that was sudden along with paracentral scotoma for 1 week. There was no history of smoking, drugs, trauma, surgery, hypotension, caffeine or fever, flu or sinus infection. Best-corrected visual acuity (BCVA) measured 20/20 OD and 20/30 OS, and intraocular pressures were within normal limits OU. Pupillary examination and anterior segment was unremarkable in both the eyes done by slit lamp biomicroscopy. Dilated fundus examination showed multiple perifoveal petalloid shaped lesion with the apex pointing towards the fovea in left eye. (Figure 1 color 
fundus photograph and infrared imaging that highlights the lesion and Figure 2 of optical coherence tomography shows hyper-reflective bands in the outer nuclear layer and outer plexiform layer, along with disruption of ellipsoid and inter-digitation zones). The right eye fundus was normal. Amsler grid showed scotoma corresponding to the macular lesion drawn by the patient and so did the Visual field of the left eye as shown in the figure $3 \mathrm{a}$ of visual field and 3 bamsler grid. Patient's consent was sought before preparation of case report.

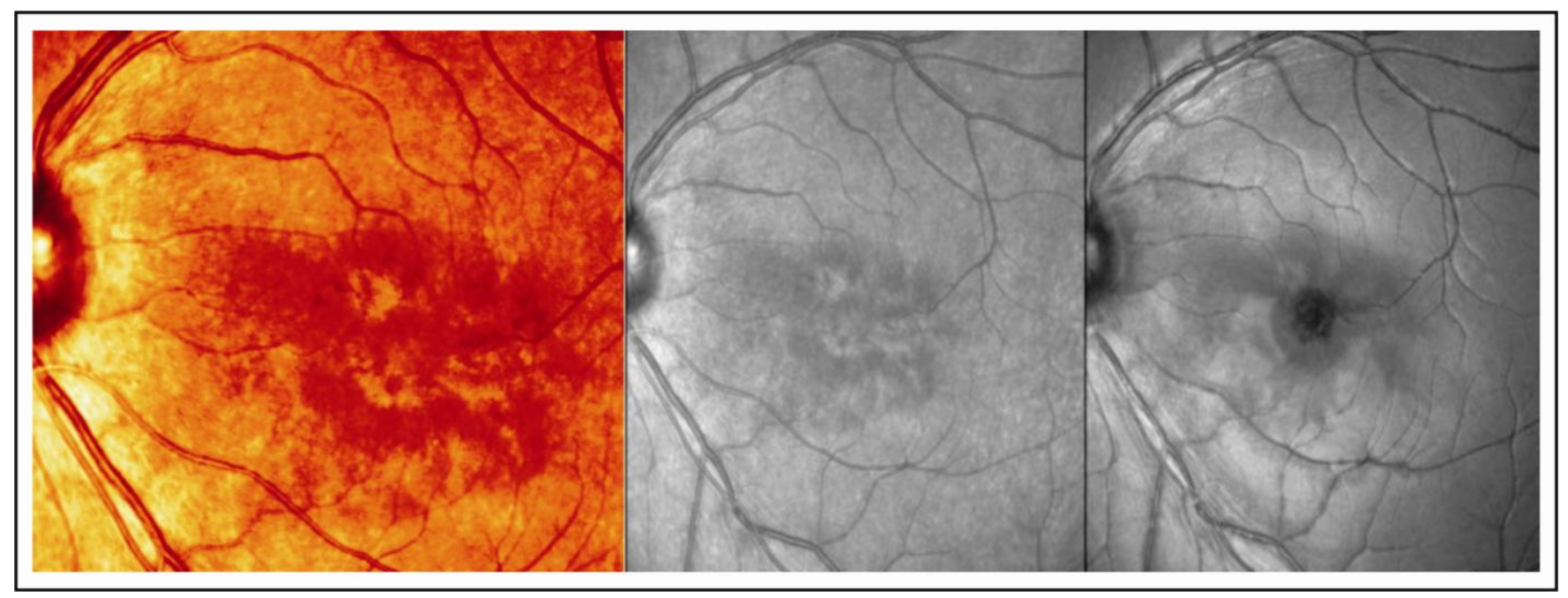

Figure 1: Color and infrared fundus photo of the left eye demonstrating a reddish brownish petalloid lesion on the macula. The right-most image shows mild recovery after 5 months of follow up.

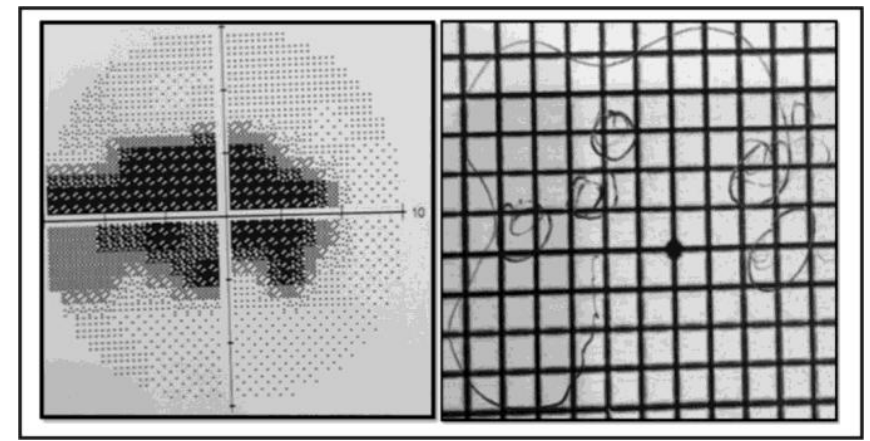

Figure 2: OCT showshyper-reflective bands in the ONL and OPL, along with disruption of ellipsoid and interdigitation zones.

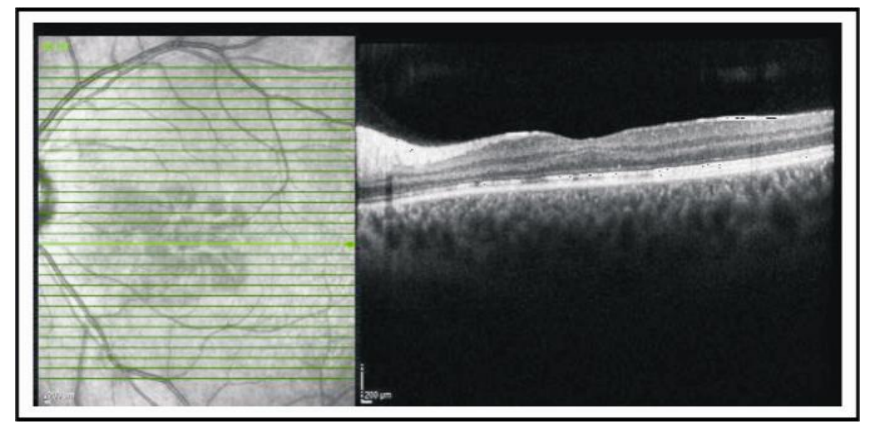

Figure 3: Visual field and Amsler grid shows paracentral lesion corresponding to the scotoma and the retinal lesion on the of figure 1.

\section{DISCUSSION}

We found the typical clinical features of AMN in our patient. Infrared clearly highlights the features of AMN in more detail. Hyper-reflective bands in the Outer nuclear layer and Outer plexiform layer with ellipsoid and interdigitation zone disruption showed that it was a classic type 1 AMN.

Earlier a case of AMN was seen in a woman using oral contraceptive pills. ${ }^{6}$ Other conditions like dengue fever, cocaine use, trauma, shock, eclampsia, epinephrine, sympathomimetic use, hypovolaemia, heavy coffee or caffeine intake and systemic lupus erythematosus are also reported to be associated with AMN.

The two types of AMN have been recently described by Rahimsy and colleagues as two separate entities. $^{8}$ The one, involving the outer retina as in our patient is considered as classic type 1 and Paracentral acute middle maculopathy PAMM type 2 based on OCT results. ${ }^{8}$ It was seen that although there are no signs of hypertensive retinopathy but our patient found to have essential hypertension. We counselled and referred the patient to the internist to control his blood pressure. However, on his second follow up visit after 
3 months, his findings remained more or less the same and scotoma persisted. That could be because of the thinning of the retinal layers/outer nuclear layer that is involved by the lesion. ${ }^{9}$

\section{CONCLUSION}

$\mathrm{AMN}$ is a rare disease entity and in our case it was associated with essential hypertension that the patient was unaware of. It is very important to diagnose rare cases using relevant investigation to save unnecessary time and money of the institute and the patient.

\section{Conflict of Interest}

Authors declared no conflict of interest.

\section{Disclaimer}

This case report has been presented as a poster in 35th Singapore Malaysia joint meeting in Ophthalmology in conjunction with 1st Asia Pacific Ocular Imaging Society Meeting 17-19th Jan 2020.

\section{REFERENCES}

1. Bos PJM, Deutman AF. Acute macular neuroretinopathy. Am J Ophthalmol. 1975; 80 (4): 573584.

2. Bhavsar KV, Lin S, Rahimy E, Joseph A, Freund KB, Sarraf D, et al. Acute macular neuroretinopathy: a comprehensive review of the literature. Surv Ophthalmology, 2016; 61 (5): 538-565.

3. Kim SE, Lee SE, Kim Y-y. A case of acute macular neuroretinopathy after non-ocular trauma. J Korean Ophthalmol. 1970; 57 (12): 1970-1975.
4. Kuriakose RK, Chin EK, Almeida DRP. An Atypical Presentation of Acute Macular Neuroretinopathy after Non-Ocular Trauma. Case Rep Ophthalmol. 2019; 10 (1): 1-4.

5. Gillies M, Sarks J, Dunlop C, Mitchell P. Traumatic retinopathy resembling acute macular neuroretinopathy. Aust N Z J Ophthalmol. 1997; 25: 207-210.

6. Kumar V, Tewari R, Yadav D, Vikas SJ. Acute macular neuroretinopathy in a young hypertensive patient. Clin Exp Optom. 2017; 100 (3): 288-290.

7. Yu S, Pang CE, Gong Y, Freund KB, Yannuzzi LA, Rahimy E, et al. The spectrum of superficial and deep capillary ischemia in retinal artery occlusion. Am J Ohthalmol. 2015; 159 (1): 53-63. e2.

8. Rahimy E, Kuehlewein L, Sadda SR, Sarraf D. Paracentral Acute Middle Maculopathy: What We Knew Then and What We Know Now. Retina. 2015; 35 (10): 1921-1930. Doi: 10.1097/IAE.0000000000000785.

9. Fawzi AA, Pappuru RR, Sarraf D, Le PP, McCannel CA, Sobrin L, et al. Acute macular neuroretinopathy: long-term insights revealed by multimodal imaging. Retina, 2012; 32 (8): 1500-1513.

\section{Authors' Designation and Contribution}

Royala Zaka; Consultant Ophthalmologist: Concepts, Design, Literature search, Data analysis, Manuscript preparation.

Yasir Khan; Consultant Ophthalmologist: Data acquisition, Statistical analysis, Manuscript editing.

Zaki-ud-din Ahmed Sabri; Consultant Ophthalmologist: Literature search, Manuscript editing, Manuscript review. 